\title{
Continuous DC-CIK Infusions Restore CD8+ Cellular Immunity, Physical Activity and Improve Clinical Efficacy in Advanced Cancer Patients Unresponsive to Conventional Treatments
}

\author{
Yan-Jie Zhao ${ }^{1 \&}$, Ni Jiang ${ }^{1 \&}$, Qing-Kun Song ${ }^{1}$, Jiang-Ping Wu ${ }^{1}$, Yu-Guang Song ${ }^{1}$, \\ Hong-Mei Zhang ${ }^{1}$, Feng Chen ${ }^{1}$, Lei Zhou ${ }^{1}$, Xiao-Li Wang ${ }^{1}$, Xin-Na Zhou ${ }^{1}$, Hua- \\ Bing Yang ${ }^{1}$, Jun Ren ${ }^{1 *}$, Herbert Kim Lyerly ${ }^{2 *}$
}

\begin{abstract}
Background: There are few choices for treatment of advanced cancer patients who do not respond to or tolerate conventional anti-cancer treatments. Therefore this study aimed to deploy the benefits and clinical efficacy of continuous dendritic cell-cytokine induced killer cell infusions in such patients. Materials and Methods: A total of 381 infusions (from 67 advanced cases recruited) were included in this study. All patients underwent peripheral blood mononuclear cell apheresis for the following cellular therapy and dendritic cells-cytokine induced killer cells were expanded in vitro. Peripheral blood $\mathrm{T}$ lymphocyte subsets were quantified through flow cytometry to address the cellular immunity status. Clinical efficacy and physical activities were evaluated by RECIST criteria and Eastern Cooperative Oncology Group scores respectively. Logistic regression model was used to estimate the association between cellular infusions and clinical benefits. $\underline{\text { Results: }}$ An average of $5.7 \pm 2.94 \times 10^{9}$ induced cells were infused each time and patients were exposed to 6 infusions. Cellular immunity was improved in that cytotoxic $\mathrm{CD8}^{+} \mathrm{CD}^{+} 8^{+} \mathrm{T}$ lymphocytes were increased by $74 \%$ and suppressive $\mathrm{CD8}^{+} \mathrm{CD28}-\mathrm{T}$ lymphocytes were elevated by $16 \%(p<0.05)$. Continuous infusion of dendritic cells-cytokine induced killer cells was associated with improvement of both patient status and cellular immunity. A median of six infusions were capable of reducing risk of progression by $70 \%(95 \%$ CI 0.10-0.91). Every elevation of one ECOG score corresponded to a 3.90-fold higher progression risk $(p<0.05)$ and $1 \%$ increase of $\mathrm{CD8}^{+} \mathrm{CD28}-\mathrm{T}$ cell proportion reflecting a $5 \%$ higher risk of progression $(p<0.05)$. Conclusions: In advanced cancer patients, continuous dendritic cell-cytokine induced killer cell infusions are capable of recovering cellular immunity, improving patient status and quality of life in those who are unresponsive to conventional cancer treatment.
\end{abstract}

Keywords: DC-CIKs - ECOG scores - cell immunity - clinical efficacy

Asian Pac J Cancer Prev, 16 (6), 2419-2423

\section{Introduction}

Cancer has become the leading cause of death in the world. According to the data of IARC (Ferlay et al., 2013), there were 14.1 million new cancer cases and 8.2 million cancer deaths in worldwide in 2012. Among them, $21.8 \%$ (3.1 million) of new cases and $26.9 \%$ (2.2 million) of deaths occurred in China. The ratio of mortality/ incidence in China is higher than the other countries $(72 \%$ vs $58.2 \%$ ). These are increasingly demands for more treatment choices for those who failed and/or untolerated to anti-cancer treatments. More and more clinical evidence showed that cancer immunotherapy is becoming one of the promising approaches addressing Eastern Cooperative
Oncology Group (ECOG) score improvements and psychological stress (Danielle et al., 2003). Our previous study indicated that for advanced cancer patients, DC-CIK immunotherapy can restore the immune status effectively (Zhao et al., 2014). The purpose of this study is to explore that whether DC-CIK treatment and the improvement of immune status can raise the clinical efficacy.

The mechanism of immunotherapies included stimulation and redirection of the cellular immune responses among cancer patients and leading to cell lysis (Choudhury et al., 2008). Dendritic cells (DCs) are the most potent antigen-presenting cells and able to promote the generation of helper $\mathrm{T}$ cells and cytotoxic $\mathrm{T}$ cells (CTLs). DC acts as effective T-cell stimulators and

${ }^{I}$ Cancer Center, Beijing Shijitan Hospital, Capital Medical University, Beijing Key Lab of Therapeutic Cancer Vaccines, Beijing, China, ${ }^{2}$ Department of Surgery, Duke University Medical Center, Durham, USA ${ }^{\circledR}$ Equal contributors *For correspondence: renjun9688@yahoo.com 
induces a tumor-specific immune response. Cytokine induced killer cells (CIKs) are ex vivo-expanded $\mathrm{T}$ lymphocytes-a subset of $\mathrm{T}$ lymphocytes with a natural killer T-cell phenotype expressing both the CD56 and the CD3 markers, present powerful non-histocompatibility complex (MHC)cytotoxicity against many tumor target cells in many trials (Heusel et al., 1994; Kagi et al., 1994; Schmidt-Wolf et al., 1996; Bradley et al., 1998).Increasing evidences suggest that dendritic cells-cytokine induced killer cells (DC-CIKs) can increase the PFS/OS and QoL of lung cancer, breast cancer and colon cancer patients (Chen et al., 2014; Wang et al., 2014b; 2014c). Our previous study also indicated that high-dose chemotherapy combined with DC-CIKs improved both progression-free and overall survival in patients with metastatic breast cancers (Ren et al., 2013).

There were some remained issues that what was the minimum amount of DC-CIKs infusions required to restore the cellular immunity and subsequently improve the clinical benefits. Whether these infusions could be adopted to those advanced patients who failed and/or untolerated to conventional treatments.

\section{Materials and Methods}

\section{Patients}

From July 2012 to July 2013, 67 patients with advanced cancer of stage IV receiving 381 DC-CIKs infusions were recruited into the study from Beijing Shijitan Hospital, Capital Medical University Cancer Center, Beijing, China. All patients failed to and could not tolerate to any conventional anti-cancer treatments due to poor life status, elderly. The inclusion criteria was included ECOG $>2$ and chemo-radiotherapy-free at least 3 months. All patients signed the informed consent form. The study was approved by the ethical committee of Beijing Shijitan Hospital. Prior and post one month of DC-CIKs infusions, peripheral blood T lymphocytes were cytometrilized with the contrast of CT or MRI imaging. RECIST criteria was used to evaluated the clinical efficacy. Meantime ECOG status was recorded periodically for physical activity assessment.

\section{ECOG and RECIST criteria}

ECOG: 0-Fully active, no restrictions on activities; 1-Unable to do strenuous activities, but able to carry out light housework and sedentary activities;2 -Able to walk and manage self-care, but unable to work. Out of bed more than $50 \%$ of waking hours; 3 -Confined to bed or a chair more than $50 \%$ of waking hours. Capable of limited selfcares; 4-Completely disabled. Totally confined to a bed or chair. Unable to do any self-care; 5-Death.

RECIST criteria: Complete response (CR): Disappearance of all target lesions. Any pathological lymph nodes (whether target or non-target) must have reduction in short axis to $<10 \mathrm{~mm}$. Partial response (PR): At least a $30 \%$ decrease in the sum of diameters of target lesions, taking as reference the baseline sum diameters. Progressive disease (PD): At least a $20 \%$ increase in the sum of diameters of target lesions, taking as reference the smallest sum on study (this Includes the baseline sum if that is the smallest on study). In addition to the relative increase of $20 \%$, the sum must also demonstrate an absolute increase of at least $5 \mathrm{~mm}$. (the appearance of one or more new lesions is also considered progression). Stable disease (SD): Neither sufficient shrinkage to qualify for PR nor sufficient increase to qualify for PD.

\section{Cell isolation and antibodies reagents}

$2 \mathrm{ml}$ of heparinized peripheral blood was drawn from each patient. Then $100 \mu \mathrm{l}$ blood was incubated in the dark with primary antibody at $4^{\circ} \mathrm{C}$ for $15 \mathrm{~min}$. After hemolysis for $10 \mathrm{~min}$, samples were centrifuged for $10 \mathrm{~min}$ at 1500 $\mathrm{rpm}$ at room temperature, and then washed twice in PBS and subjected to flowcytometric analysis (BectonDickinson, Franklin Lakes, NJ). Primary antibodies included: anti-CD4-FITC, anti-CD8-PE, anti-CD3-PerCP (Becton-Dickinson), anti-CD4-FITC (Beckman-Coulter), anti-CD25-PE (Beckman-Coulter), anti-CD28-FITC (Beckman-Coulter), anti-CD8-PE (Beckman-Coulter), anti-CD3-FITC .

\section{Flow cytometric analysis}

Flow cytometric analysis was performed to determine cell phenotypes. Lymphocyte subset levels were reported as percentages of the total population. Flow cytometry was performed using an FC500 (Beckman-Coulter), and CXP analysis software (Beckman-Coulter) was used for analysis.

DC-CIK induction, generation and infusion

For the induction of DC-CIKs, peripheral blood

Table 1. Multi-variate Analysis of Factors to the Change of ECOG and Immune Function

\begin{tabular}{|c|c|c|c|c|c|c|}
\hline & \multicolumn{2}{|c|}{ ECOG } & \multicolumn{2}{|c|}{$\begin{array}{c}\mathrm{CD} 8{ }^{+} \mathrm{CD} 28^{+} \\
\mathrm{T} \text { cell }\end{array}$} & \multicolumn{2}{|c|}{$\begin{array}{c}\mathrm{CD} 8^{+} \mathrm{CD} 28^{-} \\
\mathrm{T} \text { cell }\end{array}$} \\
\hline & B & $\bar{p}$ & B & $p$ & B & $\bar{p}$ \\
\hline DC-CIK cycles & -0.36 & 0.024 & 0.55 & 0.89 & -13.75 & 0.001 \\
\hline age & -0.12 & 0.432 & -6.34 & 0.121 & 1.22 & 0.75 \\
\hline $\operatorname{sex}$ & 0.002 & 0.65 & -0.255 & 0.06 & 0.03 & 0.83 \\
\hline
\end{tabular}

Table 2. Basic Characteristics of Controlled and Progressed Cases

\begin{tabular}{|c|c|c|c|}
\hline & Controlled cases & Progressed cases & $p$ \\
\hline Age & $63.3 \pm 15.9$ & $60.1 \pm 14.4$ & 0.397 \\
\hline \multicolumn{4}{|l|}{ Sex } \\
\hline Male & $14 \quad(50)$ & $19(48.7)$ & 0.918 \\
\hline Female & $14 \quad(50)$ & $20(51.3)$ & \\
\hline BMI & $22.7 \pm 3.95$ & $22.95 \pm 3.15$ & 0.78 \\
\hline Cancer type & & & 0.108 \\
\hline Breast & $3 \quad(25)$ & $9(75)$ & \\
\hline Lung & $5(31.3)$ & $11(68.8)$ & \\
\hline Gastric & $3 \quad(30)$ & $7 \quad(70)$ & \\
\hline Others & $17(58.6)$ & $12(41.4)$ & \\
\hline Surgery & & & 0.44 \\
\hline yes & $17(45.9)$ & $20(54.1)$ & \\
\hline no & $11(36.7)$ & $19(63.3)$ & \\
\hline Chemotherapy & & & 0.33 \\
\hline yes & $23(45.1)$ & $28(54.9)$ & \\
\hline no & $5(31.3)$ & $11(68.7)$ & \\
\hline Radiotherapy & & & 0.95 \\
\hline yes & $7(41.2)$ & $10(58.8)$ & \\
\hline no & $21 \quad(42)$ & $29 \quad(58)$ & \\
\hline
\end{tabular}


DC-CIK Infusions Restore CD8+Immunity, Physical Activity and Improve Clinical Efficacy in Advanced Cancer Patients Table 3. Therapy Managements between Controlled and Progressed Cases

\begin{tabular}{|c|c|c|c|c|c|}
\hline & Controlled cases & Progressed cases & $p$ & $\mathrm{RR}^{*}$ & $95 \% \mathrm{CI}$ \\
\hline DC-CIK cycles & & & $<0.05$ & 0.3 & $0.10-0.91$ \\
\hline 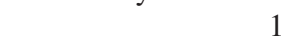 & $7(10.4)$ & $20(29.9)$ & & & \\
\hline$>1$ & 21(31.3) & $19(28.4)$ & & & \\
\hline Change of ECOG & $-0.79 \pm 0.63$ & $-0.33 \pm 0.58$ & $<0.05$ & 3.9 & $1.50-10.06$ \\
\hline Change of $\mathrm{CD}^{+} \mathrm{CD} 28^{-}$ & $-9.64 \pm 14.08$ & $-0.22 \pm 16.98$ & $<0.05$ & 1.05 & $1.01-1.09$ \\
\hline Change of $\mathrm{CD} 8^{+} \mathrm{CD} 28^{+}$ & $9.34 \pm 17.56$ & $8.76 \pm 15.53$ & 0.888 & - & \\
\hline
\end{tabular}

*Cox Hazard Proportional Model adjusting age, sex, BMI and previous treatment (surgery, chemotherapy and radiotherapy)

mononuclear cells (PBMCs) were mobilized by G-CSF. Apheresis was performed using the COBE Spectra cell separator (COBE BCT, Lakewood, CO, USA) and repeated until $\geq 4.5 \times 10^{6} / \mathrm{kg} \mathrm{CD} 34^{+}$was collected. The mean yield of $\mathrm{CD}^{2} 4^{+}$cells was $1.22 \pm 1.13 \times 10^{6} / \mathrm{kg}$. $25-50 \mathrm{ml}$ of the apheresis product was cultured in vitro to generate autologous DCs by culture in the presence of IL-4,TNFaand GM-CSF.

Mononuclear cells prepared from peripheral blood were separated by Ficoll-Hypaque centrifugation method and activated in vitro with the recombinant cytokines IL-2 at $1000 \mathrm{U} / \mathrm{ml}$ (Boehringer Mannheim, Germany), IFN- $\gamma$ at 1000U/ml (Boehringer Mannheim, Germany) and CD3 antibody at $1.7 \mu \mathrm{l} / \mathrm{ml}$ (Boehringer Mannheim, Germany) for 7-10 days.

Cells growth were observed under the microscope, and DCs phenotypes were determined by FCM of CD80, CD86, HLA-DR, CD1a and CD11c. DCs suspension contained more than $80 \%$ of CD80+CD86+ cells before infusion. CIKs express CD3 and CD56. After culture in vitro for 7-10 days DCs and CIKs were harvested and administered intravenously QOD, three times composed 1 cycle. The interval of two cycles was 1 month.

\section{Statistical analysis}

The software of SPSS 17.0 version was used in the statistical analyses. The difference of cellular immunity and ECOG score were analyzed by paired t-test between pre- and post-immunotherapy. Multiple linear regression was used to estimate the relationship between DC-CIKs infusion cycles and change of ECOG and immunity, with the adjustments of age and sex. Between controlled and progressed cases, the difference of age, BMI, change of ECOG and change of $\mathrm{T}$ cells was estimated by t-test, and the other categorical variables were estimated by chi-square test. The significant variables were further analyzed by Cox-Hazard Proportional Regression model with adjustments of age, sex, BMI and conventional treatments. All tests were 2-sided and the significant level was set as 0.05 .

\section{Results}

\section{Cellular immunity improvement}

Average $5.7 \pm 2.94 \times 10^{9}$ of induced cells were infused each time and patients were exposed to 6 infusions. After DC-CIKs infusions, cellular immunity predominant of $\mathrm{T}$ lymphocyte was restored: the proportion of $\mathrm{CD}^{+} \mathrm{T}$ cell increased by $18.3 \%$, whereas cytotoxic $\mathrm{CD} 8{ }^{+} \mathrm{CD} 28^{+} \mathrm{T}$ cell increased significantly from $12.2 \%$ to $21.2 \%$ with the contrast to suppressive $\mathrm{CD} 8^{+} \mathrm{CD} 28^{-} \mathrm{T}$ cell reduced by
$15.8 \%$. Every addititional three infusions were related with reduction of $\mathrm{CD}^{+} \mathrm{CD} 28^{-} \mathrm{T}$ cell proportion by $13.75 \%$ (Table 1, $p<0.05$ ).

\section{ECOG score improvement}

With DC-CIKs infusions, patients' physical status was also improved significantly and ECOG score decreased by $15.6 \%(p<0.05$, Table 1$)$. Every additional three infusions were associated with the reduction of ECOG score by 0.36 (Table 1, $p<0.05$ ).

\section{Clinical efficacy improvement}

All subjects were exposure to an average of 6 infusions of DC-CIKs. 2 patients (3.0\%) reached PR, 26 patients $(38.8 \%)$ remained stable, 39 cases $(58.2 \%)$ progressed, and the disease control rate (DCR) was $41.8 \%$. The celluar immunity recovery induced by DC-CIKs infusions were not related with patients' age, gender, BMI, cancer subtype and previous treatments $(p>0.05$, Table 2$)$.

DC-CIKs infusions were inversely related with disease progression, six and above infusions might reduce the risk by $70 \%(\mathrm{HR}=0.30,95 \% \mathrm{CI} 0.10-0.91)$ (Table 3$)$. Elevation of ECOG score and $\mathrm{CD} 8^{+} \mathrm{CD} 28 \mathrm{~T}$ cells proportion were associated with higher risk of disease progression: every elevation of one ECOG score corresponded to a 3.90fold higher progression risk $(p<0.05)$ and $1 \%$ increase of $\mathrm{CD}{ }^{+} \mathrm{CD} 28^{-} \mathrm{T}$ cell proportion reflecting a $5 \%$ higher risk of progression $(p<0.05)$ (Table 3$)$.

\section{Adverse reactions}

There were no obvious side effects occurred during the entire infusions except that two patients presented a transient mild fever and chill.

\section{Discussion}

Cancer immunotherapy has become a frontier approach in the last 5 years, convincing evidences come out of several clinical trials. The clinical benefits were seen from the previous studies, regarding to lung cancer (Han et al., 2014; Jin et al., 2014; Shi et al., 2014), breast cancer (Ren et al., 2013), renal cancer (Wang et al., 2014a) and gastrointestinal cancer (Shi et al., 2012; Gao et al., 2014; Wang et al., 2014b) etc. DCs have been proven as the most effective antigen-presenting cells, which can digest by endocytose antigens including circulating tumorassociated antigens to elicit the general immune responses and diminish T cell exhausting (Liu et al., 2009; Zhao et al., 2014). Due to cancer heterogeneity and randomized mutiple gene mutations, tumor microenvironment limited some anti-cancer activities induced by chemo and radio 
therapy. DC-CIKs infusions were capable of removing immuno suppression conditions. Meanwhile, some cytokines including gamma interferon and IL-12 were also increased (Marten et al., 2001).

Peripheral blood different lymphocyties distribution could represent humoral celluar immunity, even though pathological analysis was not eligible. Our previous study has showed that an increase in the percentage of the $\mathrm{CD} 8^{+} \mathrm{CD} 28$ - lymphocyte subset from the peripheral blood of metastatic breast cancer patients accompanied by increased IL- 6 and IL-10, the elevated CD8+CD28associated with shorten PFS (Song et al., 2013). Clinical results showed that with continous DC-CIK infusions alone, the ratio of $\mathrm{CD} 8^{+} \mathrm{CD} 28^{-} \mathrm{T}$ cells were reduced and the immune status were improved for advanced cancer patients (Zhao et al., 2014). Even in the situation of chemotherapy exposure, DC-CIKs infusions could synchronize chemotherapy ability (Ren et al., 2013). In this study, we explored the relationship of DC-CIKs infusions with the restoring of celluar immuity through quantitative analysis. Our data showed that with the addition of DC-CIKs infusions, the overall ECOG status was dramatically improved, every additional 3 infusions were associated with the reduction of ECOG score by 0.36 (see Table 2). Interestingly, either ECOG score or $\mathrm{CD} 8^{+} \mathrm{CD} 28^{-} \mathrm{T}$ cell proportion was increaed, the patients underwent a higher risk of disease progression.

The amount of DC-CIKs cells plays a key role in restoring cellular immunity. The removal of suppressive activities resulted from cancer cell and microenvironment has become the major interesting when anti-cancer treatment employed in vivo. Inadequate cell infusion was not capable of diminishing immunity suppression. We postulated that provision of minimum DC-CIKs was clinically required to conduct the effective immune responses. In this study, we calculated the average infusion cells number of $5.7 \times 10^{9}$ as the basic requirement. Othewise some invalid infusions may occur, which explain the bias of incapability of immunotherapy in some trials. We have not observed the hyper immune reactivity which might be a potential risk when exposure to repetitive DCCIKs infusions. The validation of monitoring peripheral blood cellular immunity network in vitro through $\mathrm{T}$ lymphocytes has also be useful for those patients who undertaken the secondary cancer or immunosuppressive reagent exposure.

In conclusion, for patients failed and untolerated to conventional anti-cancer treatments, continuous DCCIKs infusions could restore cellular immunity, improve the patients physical status and reduce the risk of disease progression. The essential amount of DC-CIK cells was addressed.

\section{Acknowledgements}

We are indebt to all patients recruited in this study to cooperate the blood collections, cell apheresis and data collections.

\section{Financial support}

Special funds of the science and technology innovation base for Beijing key laboratory of therapeutic cancer vaccine (No.Z131102002813059). Beijing Municipal Administration of Hospitals Clinical Medicine Development of Special Funding Support, code: XMLX201413.

\section{References}

Bradley M, Zeytun A, Rafi-Janajreh A, et al (1998). Role of spontaneous and interleukin-2-induced natural killer cell activity in the cytotoxicity and rejection of Fas+ and Fastumor cells. Blood, 92, 4248-55.

Chen R, Deng X, Wu H, et al (2014). Combined immunotherapy with dendritic cells and cytokine-induced killer cells for malignant tumors: a systematic review and meta-analysis. Int Immunopharmacol, 22, 451-64.

Choudhury A, Palma M, Mellstedt H (2008). The future of cancer vaccines for non-small-cell lung cancer: ongoing trials. Clin Lung Cancer, 9, 37-44.

Danielle D, Parker P, Fouladi R, et al (2003). The association between social support, intrusive thoughts, avoidance, and adjustment following an experimental cancer treatment. Psychooncology, 12, 453-62.

Ferlay J, Soerjomataram I, Ervik M, et al (2013). GLOBOCAN 2012 v1.0, cancer incidence and mortality worldwide: IARC CancerBase No. 11 (Internet). Lyon, France: international agency for research on cancer; Available from: http:// globocan.iarc.fr, accessed on day/month/year.

Gao D, Li C, Xie X, et al (2014). Autologous tumor lysate-pulsed dendritic cell immunotherapy with cytokine-induced killer cells improves survival in gastric and colorectal cancer patients. PLoS One, 9, 93886.

Han RX, Liu X, Pan P, et al (2014). Effectiveness and safety of chemotherapy combined with dendritic cells co-cultured with cytokine-induced killer cells in the treatment of advanced non-small-cell lung cancer: a systematic review and meta-analysis. PLoS One, 9, 108958.

Heusel J, Wesselschmidt R, Shresta S, et al (1994). Cytotoxic lymphocytes require granzymeB for the rapid induction of DNA fragmentation and apoptosis in allogeneic target cells. Cell, 76, 977-87.

Jin C, Li J, Wang Y, et al (2014). Impact of cellular immune function on prognosis of lung cancer patients after cytokineinduced killer cell therapy. Asian Pac J Cancer Prev, 15, 6009-14.

Kagi D, Ledermann B, Burki K, et al (1994). Cytotoxicity mediated by $\mathrm{T}$ cells and natural killer cells is greatly impaired inperforin-deficient mice. Nature, 5, 31-7.

Liu P, Chen L, Huang X (2009). The antitumor effects of CIK cells combined with docetaxel against drug-resistant lung adenocarcinoma cell line SPC-A1/DTX in vitro and in vivo. Cancer Biother Radiopharm, 24, 91-8.

Märten A, Ziske C, Schottker B, et al (2001). Interactions between dendritic cells and cytokine-induced killer cells lead to an activation of both populations. J Immunother, 24, 502-10.

Ren J, Di L, Song G, et al (2013). Selections of appropriate regimen of high-dose chemotherapy combined with adoptive cellular therapy with dendritic and cytokine-induced killer cells improved progression-free and overall survival in patients with metastatic breast cancer: reargument of such contentious therapeutic preferences. Clin Transl Oncol, 15, 780-8.

Schmidt-Wolf IG, Lefterova P, Johnston V, et al (1996). Sensitivity of multidrug-resistant tumor cell lines to 
DC-CIK Infusions Restore CD8+Immunity, Physical Activity and Improve Clinical Efficacy in Advanced Cancer Patients immunologic effector cells. Cell Immunol, 169, 85-90.

Shi L, Zhou Q, Wu J, et al (2012). Efficacy of adjuvant immunotherapy with cytokine-induced killer cells in patients with locally advanced gastric cancer. Cancer Immunol Immunother, 61, 2251-9.

Shi SB, Tang XY, Tian J, et al (2014). Efficacy of erlotinib plus dendritic cells and cytokine-induced killer cells in maintenance therapy of advanced non-small cell lung cancer. J Immunother, 37, 250-5.

Song G, Wang X, Jia J, et al (2013). Elevated level of peripheral CD8(+)CD28(-) T lymphocytes are an independent predictor of progression-free survival in patients with metastatic breast cancer during the course of chemotherapy. Cancer Immunol Immunother, 62, 1123-30.

Wang D, Zhang B, Gao H, et al (2014a). Clinical research of genetically modified dendritic cells in combination with cytokine-induced killer cell treatment in advanced renal cancer. BMC Cancer, 14, 251.

Wang ZX, Cao JX, Liu ZP, et al (2014b). Combination of chemotherapy and immunotherapy for colon cancer in China: a meta-analysis. World J Gastroenterol, 20, 1095-106.

Wang ZX, Cao JX, Wang M, et al (2014c). Adoptive cellular immunotherapy for the treatment of patients with breast cancer: a meta-analysis. Cytotherapy, 16, 934-45.

Zhao Y, Song Y, Zhang H, et al (2014). Clinical analysis of patients suffering from advanced malignancy with survival time less than 3 months by DC-CIK cells. Cancer Res Prev Treat, 41, 397-400. 